\title{
Multidisciplinary Collaborative Optimization Design for Oil Tanker Concept Design
}

\author{
Song Du \\ Ship CAD Engineering Center, Dalian University of \\ Technology, Dalian, Liaoning, China \\ dusong_2002@163.com
}

\author{
Yan Lin \\ Ship CAD Engineering Center, Dalian University of \\ Technology, Dalian, Liaoning, China \\ linyanly@dlut.edu.cn
}

\begin{abstract}
The idea of multidisciplinary design optimization is applied to the solution of the optimization design of oil tankers. The multidisciplinary design optimization model is established according to the multi-objective and multi-constraints characteristics of the design of oil tanker. The optimization model is solved by using the collaborative optimization algorithm, and its solution is completed. The numerical results indicate that the collaborative optimization can be successfully applied to dealing with the efficiency of the optimization design as well as the problems of an oil tanker, which lays a foundation to solve more complex engineering systems.
\end{abstract}

Keywords-Multidisciplinary design optimization; concept design; collaborative optimization; oil tanker; EEDI

\section{INTRODUCTION}

Multidisciplinary design optimization (MDO) ${ }^{[1]}$ may be the potential development trends for the research and industrial application in ship field. Despite the not very long time for the research of MDO in ship field $d^{[2,3]}$, it has been realized that MDO applied in the relevant ship-developing process could improve the quality and efficiency of ship design as well as play a decisive role in the future ship design field.

The design of a ship is a typical issue featuring interdisciplinary coupling which touches upon the optimization of integrating many factors like the ship's economy, technical performance, beauty and comfort as well as the navigation safety and environment protection. However, the design of a ship is not a simple accumulation of the designs of the various subsystems, but an interactive and mutually restricting relation in applying various disciplines. On the premise of how to efficiently take these coupling relations into account and how to acquire the optimal comprehensive interests are long-term research works that the ship designers are faced with. The design of a ship will be abstracted as the designs of various disciplines by reasonable decomposition, the mathematical models of ships featuring the multidisciplinary co-design will be established and the various disciplines will be analyzed and solved by adopting effective methods and strategies.

Although the profit of optimizing the scheme of hull form merely accounts for 3\%-5\% of the total costs, the risks and workload referred is correspondingly the lowest and the rework rate will be reduced effectively. Traditionally, the scheme of ship design is demonstrated from the economic and technical perspectives in terms of the key elements of hull form. However, this paper introduces new Ship Energy and Efficiency Design Index (EEDI) ${ }^{[4]}$ as a consideration factor on the basis of the demonstration of the ship design from the economic and technology perspective and adopts the idea of multidisciplinary design to construct new demonstration methods of the scheme optimization of hull form as well as applies the strategy of Collaborative Optimization (CO) to demonstrate the design of oil tankers, which turns out to be satisfactory.

\section{COLLABORATIVE OPTIMIZATION}

\section{A. The Basic Ideas of Collaborative Optimization}

Proposed by Kroo, Braun et al. of Stanford University through the Compatibility Constraint Optimization Algorithm, the strategy of Collaborative Optimization is a MDO method featuring bi-level optimization structures ${ }^{[5] \text {. }}$ Among the various MDO algorithms, CO algorithm is a popular one. According to the $\mathrm{CO}$ algorithm, the original design optimization is decomposed into a systematic optimization and multidisciplinary optimization and by regarding all the state vectors as design vectors, the disciplinary coupling relations are lifted and the multidisciplinary optimization could be independently and paralleled achieved.

CO algorithm makes the MDO issue decomposed into a bi-level hierarchy optimization structure. The top level is a systematic optimization module to optimize the multidisciplinary variables. The systematic optimization module aims at minimizing the systematic aims and satisfying the interdisciplinary constraints. The sub-system optimization module conducts optimization by taking these factors as its aims, like the calculations through the analysis of the subsystems and the minimal variance of the variable subsets of the subsystem design. While conducting optimization, the systematic design variables are considered as fixed values, whereas the interdisciplinary cross variables and disciplinary design variables are set in the analysis of the subsystems. As a rule, to cope with the multidisciplinary compatible constraint, inequality is adopted. In CO algorithm, the subsystems aim at the results of the relevant disciplinary design optimization and the minimization of scheme differences provided by the systematic level. Therefore, the multidisciplinary coupling variables are not necessarily coordinated by the systematic level and the optimal design scheme could be formulated by multiple iterations. 


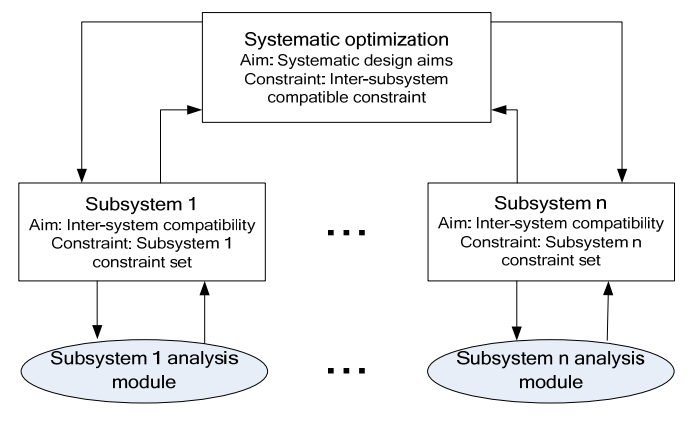

Figure 1. The basic framework of Collaborative Optimization

\section{B. The Solving Process of Collaborative Optimization}

The basic solving procedures are as following, shown in figure 2:

Step1. The ideal solutions are calculated to construct the mathematic model of systematic optimization;

Step2. The initial value of design variables are adopted by the systematic level to acquire the initial coordinate value of the instrumental variable $\mathrm{Z}$ (systematic variable) which is then delivered to the subsystems;

Step3. After acquiring the initial coordinate value, the instrumental variables ${ }^{-j i}$ and the compatible constraint of the relevant variables are introduced into the various disciplines. Meanwhile, the independent variable $\mathrm{xi}$, the systematic variable $\mathrm{x}$ and the instrumental variable $\bar{y}^{-j i}$ are solved;

Step4. The optimal value is sent back from the subsystems to the systematic level. Then, the linear combination is conducted by the systematic level to eliminate the incompatibility and the solution $\mathrm{Z}$ is obtained in systematic level;

Step5. The comparison between this value and the design variable value previously sent to the subsystems is made. If the difference lies in an acceptable range, the iteration will be finished. Or else, go on to the Step 6;

Step6. After the new coordinate $\mathrm{Z}$ is calculated, the variables, previously calculated by the various disciplines, are regarded as the initial value and then the solution is preceded. Afterwards, turn to Step3.

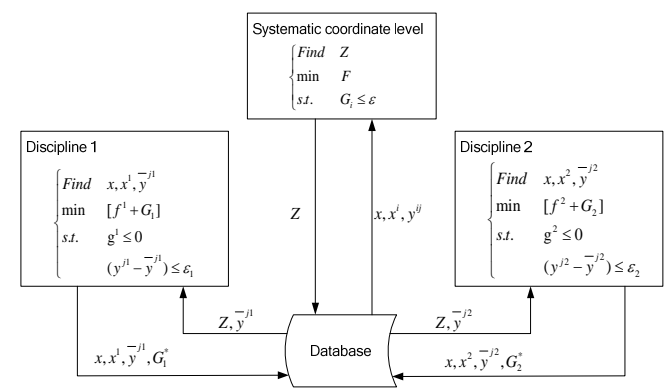

Figure 2. Optimization model of CO

\section{MultidisciplinARY COLLABORATIVE TANKER DESIGN}

\section{A. The Multidisciplinary Design Optimization model of Oil Tanker Design}

The core of ship design is the determination of the principal dimension, the ship form index and the main engine, which lays a foundation for linear design and general layout design. To start with, according to the concrete requirements of the task, the ship MDO systematic model, consisting of the three features like technical performance, economic performance and energy efficiency level, is built. Among these features, technical performance mainly takes navigational speed, stability and rolling period as the optimized objectives, the economic performance primarily takes the initial cost, the basic freight rate, payback period and net present value as its objectives and the energy efficiency level takes EEDI as its objective. This model adopts hierarchic multidisciplinary model. Concretely, to meet the requirements of the task and the restrictions with respect to the shipyard, the slipway and the dock, the constraint conditions are formulated, including the design variable interval and the upper and lower bounds of some state variables. Plus, the ship MDO model, reflecting the multidisciplinary parallel design, is established.

The procedures to build the MDO model of ship design are shown as:

Step1. Determine the objective function at the systematic level with the pursuit of the comprehensive optimal scheme integrating the ship's technical performance, economic performance and environmental protection.

Step2. Determine the constraint conditions at systematic level in accordance with the ship dimensions, the requirements of the ship owner and safety considerations.

Step3. Determine the objective functions of the subsystem designs by coming up with a mathematic formula in accordance with the design norms and requirements of the subordinate disciplines along with the decomposition of tasks.

Step4. Determine the exchange relations between the variables and the data. The values of independent variables could be determined in the sub-level tasks while the determination of the coupling variables must be conducted collaboratively and the design requirements of the relevant disciplines should be expressed in constraint conditions. In addition, the state variables refer to the analytic parameters of the subsystems through the analysis of the various disciplines.

\section{B. Systematic Model}

The termination standards at the systematic level:

1. Design the objective satisfaction by reaching the set value in the satisfaction function $\mathrm{F}(\mathrm{X})$ at the systematic level;

2 . When the difference of the parameter vectors obtained after the optimization of two sub systems is less than a fixed value;

3. The frequency of the systematic collaborative optimization is more than a set frequency. 
find Lpp, B, T, Cb, MRC

(1)

$$
\begin{gathered}
\max F(X)=\omega_{1} A_{1}^{\prime}(X)+\omega_{2} A_{2}^{\prime}(X)+\omega_{3} A_{3}^{\prime}(X) \\
\text { s.t. } \quad g_{j}(X) \leq 0 \quad j=1, \cdots, p \\
I_{E E D I}=3.1144 \times \frac{S F C_{M E} \square \sum_{i=1}^{n M E} P_{M E(i)}+S F C_{A E} \square P_{A E}}{C_{a p} \backslash V_{r e f} \square f_{w}}
\end{gathered}
$$

The interdisciplinary coupling relations as the systematic level are shown in figure 3:

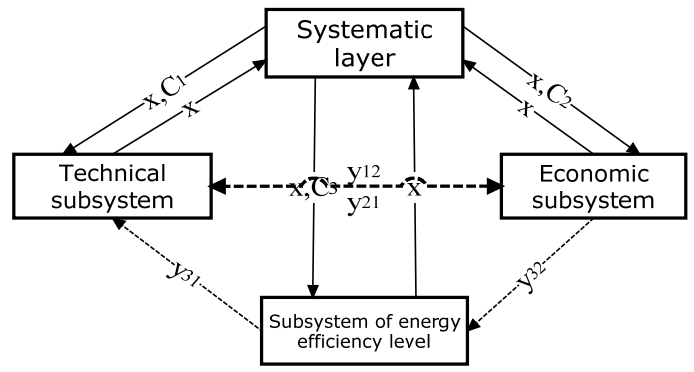

Figure 3. Disciplines coupling relationship

\section{AN APPLICATION EXAMPLE}

In accordance with the built MDO model of ship design, a 50000DWT oil tanker is selected as a data context and the MDO strategy is applied to conduct an optimized analysis and calculation of the cased design data. In the light of the general design specification of 50000DWT oil tankers, the length $\left(L_{p p}\right)$ is $186 \mathrm{~m}$, the beam $(B)$ is $34 \mathrm{~m}$, the draft $(T)$ is $12.5 \mathrm{~m}$, the block coefficient $\left(C_{b}\right)$ is 0.8168 and the engine power $(M R C)$ is $9480 \mathrm{~kW}$, the capacity tonnage $D W$ is $52825 \mathrm{t}$ and the navigational speed $V_{s}$ is $14.4837 \mathrm{kn}$. The initial value of the systematic variable in correspondence with the general design specification of the oil tanker is: $X^{0}=$ $(186,34,12.5,0.8168,9480)^{\mathrm{T}}$. In figure 4 , the model of optimization using the collaborative optimization strategy, as far as possible to ensure discipline level of satisfaction and the consistency condition of satisfaction at the same time to achieve the optimal value, in the process of collaborative optimization, to make the coupling variables and the aim have a relatively consistent between disciplines.

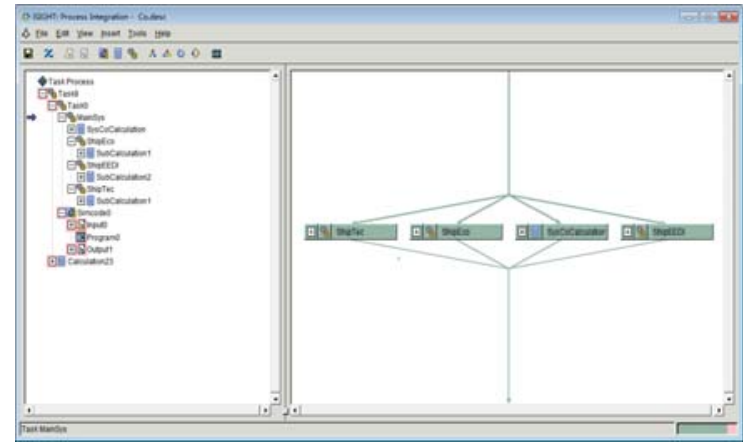

Figure 4. The simulation CO process flow for ship MDO

With regard to the different design aims of the designed oil tanker, three sets of numerical data are selected from the weight value parameters both at systematic level and at disciplinary level. As to the selection of weight, the first group of weight value parameters places weight on technical and economic balance while the second group model value parameters emphasizes the reduction of energy efficiency index. And the third group of weigh value parameters represents the objective of the ship design scheme in pursuit of high economic benefits. The CO strategy is adopted to seek the solution of the MDO ship model based on three disciplines. Three groups of weight value parameters are set to meet the design requirements, correspondingly, three sets of form model schemes for the 50000DWT oil tanker are shaped, specifically shown in TABLE I , the systematic objective values respectively increased by $3.734 \%$, 8.916\%, and $7.93 \%$ after the optimization of the systematic objective function. The optimization of the three groups of weight values brings about an ideal result.

TABLE I. COMPARISON OF PRINCIPAL DIMENSIONS

\begin{tabular}{c|c|c|c|c}
\hline & $\begin{array}{c}\text { Initial } \\
\text { Scheme }\end{array}$ & $\begin{array}{c}\text { First } \\
\text { Group }\end{array}$ & $\begin{array}{c}\text { Second } \\
\text { Group }\end{array}$ & $\begin{array}{c}\text { Third } \\
\text { Group }\end{array}$ \\
\hline $\mathbf{L}_{\mathrm{pp}}(\mathrm{m})$ & 186 & 181.5 & 191 & 182 \\
B(m) & 34 & 35.4 & 34.5 & 32.7 \\
$\mathrm{~T}(\mathrm{~m})$ & 12.5 & 12.8 & 12.7 & 12.35 \\
$\mathrm{C}_{\mathbf{b}}$ & 0.8168 & 0.8231 & 0.8243 & 0.8145 \\
MRC(kW) & 9480 & 9550 & 9620 & 9150 \\
DW(t) & 52825 & 51704 & 52939 & 48345 \\
\hline
\end{tabular}

TABLE II. COMPARISON OF PERFORMANCE

\begin{tabular}{c|c|c|c|c}
\hline & $\begin{array}{c}\text { Initial } \\
\text { Scheme }\end{array}$ & $\begin{array}{c}\text { First } \\
\text { Group }\end{array}$ & $\begin{array}{c}\text { Second } \\
\text { Group }\end{array}$ & $\begin{array}{c}\text { Third } \\
\text { Group }\end{array}$ \\
\hline V $_{\mathrm{s}}(\mathrm{kn})$ & 14.48 & 14.31 & 13.81 & 14.44 \\
GM(m) & 3.258 & 4.005 & 3.8203 & 2.6203 \\
$\mathrm{~T}_{\mathrm{s}}(\mathrm{s})$ & 12.55 & 11.024 & 11.826 & 14.294 \\
PBP(y) & 11.28 & 10.38 & 11.745 & 9.9409 \\
RFR(yuan/t) & 61.3 & 59.76 & 65.4 & 59.0 \\
P(10000yuan) & 31732.06 & 32813.42 & 33272.35 & 29029.15 \\
NPV & 18612.68 & 21677.62 & 20932.29 & 19676.79 \\
$(10000 y u a n)$ & 6.3148 & 5.838 & 5.2171 & 6.6154 \\
IEEDI & \multicolumn{4}{|l}{}
\end{tabular}


From the TABLE II , the main indicators of the hull form are demonstrated separately in correspondence to the three sets of form schemes. It is shown that the aim of the first set of form scheme is seeking the balance between the technical performance and economic performance. As a result, all the indexes gained by comparing the initial scheme and other two sets of schemes lie in the middle, which means that along with the ship energy efficiency index up to the standard, the ship's technical performance index and economic performance index is relatively balanced. The second set of form scheme offers a lower new ship energy efficiency index yet the highest cost and the longest payback period in comparison with other two, which is identical to the statistic results of EEDI. As for the third set of form scheme, aiming at pursuing a better economic performance, this set of scheme not only provides the shortest payback period, but also achieves the highest EEDI values.

From the case of 50000DWT oil tanker, the conclusion could be drawn: the application of MDO model could be helpful to work out the optimal ship form scheme in the scheme design phrase in accordance with the demands of ship designers as well as add up some reliable references for design decision. As far as the selection of weight, the first set of ship form scheme attaches importance to the balance between the rapidity and economy. Therefore, the form scheme is optimized with a higher engine power, while the second set of form scheme favors the reduction of EEDI, which makes this scheme a lowers navigational speed, a lower engine power and a higher loading capacity with conformity to the guiding thought of the new ship EEDI. And the third set of form scheme reflects the pursuit of the highest economic benefits. However, the value of EEDI in the third set is relatively high, in excess of the baseline value by $5 \%$, becomes the highest EEDI value acceptable in the model, which reveals that currently, there are still contradictions between the economic benefits and environmental protection.

\section{SUMMARY}

The optimization of the principal ship characteristics at the initial stage of ship design lays a foundation for the determination of the ship form scheme. For many years, the research in this regard has been continually conducted. It's acceptable and necessary to take the ship MDO as a touching point. This paper apply the MDO concept and construct MDO model of ship scheme design by integrating the technology, economy and environmental protection as well as select different weigh coefficients in accordance with the designer's basic assessment of the current ship form, adopt CO strategy to obtain the satisfactory design scheme and make a proof of the feasibility of MDO model by taking the oil tanker as a case study. As an application and exploration in ship design, the adoption of MDO technology to solve the issue of the optimization of the ship scheme design calls for massive in-depth work.

\section{REFERENCES}

[1] J Sobieszczanski-Sobieski,R T Haftka. Multidisciplinary aerospace design optimization: Survey of Recent Developments [C].34th AIAA Aerospace Sciences Meeting and Exhibit,Reno,Nevada,1996

[2] Hart, C.G., Vlahopoulos. A Multidisciplinary Design Optimization Approach to Relating Affordability and Performance in a Conceptual Submarine Design[D]. University of Michigan, 2009

[3] DEMKO D. Tools for Multi-Objective and Multi-Disciplinary Optimization in Naval Ship Design[D]. Virginia Polytechnic Institute and State University, 2005.

[4] MEPC.1/Circ.681,Interim Guidelines on the Method of Calculation of the Energy Efficiency Design Index for New Ships[S]. 2009.

[5] Kroo I,Aitus S,Braun R,et al.Multidisciplinary Optimization Methods for Aircraft Preliminary Design [R].AIAA Paper 94-4325-cp,1994 